Objectives Longlasting sitting position has often been associated with the discomfort and/or pain in neck-shoulder region. The objective of this study was to examine the trapezius muscle parameters among sitting and standing workers and to investigate which is more overreaching to trapezius muscle.

Methods A total of 42 female workers in two different companies were recruited in this study. There were 21 workers (sitting workers) who were sitting at the computer most of the working day and 21 (standing workers) who were standing at their workstation and assembling the products. Trapezius muscle mechanical charecteritics were measured by myotonometry, which calculates objective parameters on state of muscle mechanical properties (frequency which characterising tone, decrement which characterising elasticity and stiffness). The upper trapezius muscle was measured at resting position in seated, both on the left and right side. Also the visual analogue scale (VAS) was used to assess the intensity of pain in neck and shoulder region. The differences between two groups were assessed with indenpendent group T-test.

Results The mean neck and shoulder pain VAS score among standing workers was 1.79 (SD 2.36) and 1.36 (SD 2.35) and among sitting workers 2.40 (SD 2.75) and 2.17 (SD 2.52) respectively. The measured outcomes showed that frequency in the right side was $15.5 \%$ and in the left side $14.9 \%$ higher among sitting workers. Stiffness was also higher among sitting workers respectively $22.6 \%$ and $25.5 \%$. Trapezius muscle frequency and stiffness were also statistically higher in sitting workers ( $t$-test $p \leq 0.001)$ and in both body sites. There were no differences in decrement.

Conclusions The result showed that sitting work induces higher tone and stiffness in upper trapezius muscle than standing work. Considering that the ergonomic workplace reorganisation should be done.

\section{PREVALENCE OF MUSCULOSKELETAL DISORDERS AMONG US EMPLOYEES OF A LARGE COMPANY}

Q Le, Kreckmann, Starks, J-Morel. E. I. du Pont de Nemours and Company, Newark, United States of America

\subsection{6/oemed-2013-101717.92}

Objectives Musculoskeletal disorders (MSDs) are a leading cause of work absenteeism. Risk for MSD is multi-dimensional, and individuals with MSDs have a diverse array of co-morbid mental and physical illnesses that impact productivity in the work place. We examined the prevalence of MSDs, including low back pain (LBP), among US-based workers for a large manufacturing company.

Methods Health insurance claims data for 25,419 employees from 2006 through 2011 were used to determine eligibility and outcome status. $92 \%$ of the workforce is enrolled in a companysponsored health insurance plan. Employees were categorised as ever having any MSD according to the presence of at least one insurance claim for a list of conditions based on ICD-9 codes. Prevalence of and trends in MSD rates were evaluated for demographic and occupational characteristics.

Results Eligible workers were predominantly white (73\%) and male (75\%). Median age was 50 years old. The annual average proportion of employees who had at least one MSD insurance claim was $36 \%$. More than $33 \%$ of the MSD claims were for LBP. Female employees were more likely to have an MSDrelated insurance claim than males $(\mathrm{p}<0.01)$. The annual prevalence rate was consistent during the eligibility period, and a strong linear trend between age and any MSD-related claim was observed $(\mathrm{p}<0.01)$.
Conclusions The findings of this analysis highlight the magnitude of MSD prevalence for an ageing workforce. The prevalence of MSDs based on insurance claims was consistent with rates reported by the US Bureau of Labour Statistics for MSDs involving work absences from 1992 to 2007 (29 to 34\%). The claims-based prevalence for this workforce was lower than the self-reported proportion of MSD for the U. S. adult population $(48 \%)$. These results will inform identification and management of occupational conditions that address the impact of MSDs on work productivity.

\section{Session: L. RICOH}

\section{REVIEW OF THE MALE REPRODUCTIVE HEALTH EFFECTS OF HORMONALLY ACTIVE CONVENTIONAL AGRICULTURAL PESTICIDES USED IN SOUTH AFRICA}

${ }^{1} \mathrm{M}$ A Dalvie, ${ }^{2} \mathrm{R}$ English. 'University of Cape Town, Cape Town, South Africa; ${ }^{2}$ Health Sytems Trust, Cape Town, South Africa

\subsection{6/oemed-2013-101717.93}

Objectives The objective of this literature review was to examine and present evidence on male reproductive health effects of hormonally active conventional agricultural pesticides used in South Africa in order evaluate the need for research in this area especially in the Western Cape, a major agricultural area in the country.

Methods The literature review included electronic and paper sources of information using PubMED/MEDLINE, EBSCO, Google Scholar and The Cochrane Library as well as theses through the University of Cape Town Medical Library. Key words for the searches included pesticides, male reproduction, endocrine disruption, farm workers, farm residents and rural residents. Data from both animal studies and epidemiological studies including all study designs and countries were considered.

Results The review indentified a number $(\mathrm{n}=11)$ of contemporary-use agricultural pesticides that have been shown to induce in vitro endocrine activity and/or have been shown to affect gonadotrophin and steroid hormone release as well as male reproductive development in animals or humans. These pesticides include chlorpyrifos, cypermethrin, endosulfan, deltamethrin, dichlorvos, DNOC, fenvelerate, glyphosate, iprodione, parathion and procloraz. Rural residents in the Western Cape especially those living on farms including children are highly exposed occupationally and non-occupationally to pesticides through a number of routes. There are, however, few epidemiological studies that have investigated male reproductive health effects in humans consequent to environmental exposure to conventional agricultural pesticides and only two in South Africa. There are no longitudinal studies.

Conclusions More epidemiological studies, especially longitudinal investigations of specific pesticides in highly exposed workers and residents especially boys in settings such as the Western Cape in South Africa are required.

\section{MALE HORMONAL PROFILE TO WORKERS EXPOSED TO TOLUENE IN A PACKAGING PLANT INDUSTRIAL IN MEXICO CITY}

${ }^{1} \mathrm{G}$ M F Miranda, ${ }^{2} \mathrm{M}$ P P R Paz-Roman, ${ }^{3} \mathrm{G}$ A M Aguilar-Madrid, ${ }^{3} \mathrm{C}$ A J P Juarez-Perez, ${ }^{3}$ L B A Basurto-Acevedo, ${ }^{3} \mathrm{~L}$ H G Haro-Garcia. ${ }^{1}$ PEMEX, Venustiano Carranza, DF, Mexico; ${ }^{2}$ UNAM, Mexico, DF, Mexico; ${ }^{3}$ MSS, Mexico, DF, Mexico

10.1136/oemed-2013-101717.94 